\title{
The LAMOST spectroscopic survey of planetary nebulae in M31 and M33
}

\author{
Maosheng Xiang ${ }^{1}$, Xiaowei Liu ${ }^{2}$, Meng Zhang ${ }^{2}$, Haibo Yuan ${ }^{3}$ and \\ Zhiying Huo ${ }^{1}$ \\ ${ }^{1}$ National Astronomical Observatories, Chinese Academy of Science, China \\ email: msxiang@nao.cas.cn \\ ${ }^{2}$ Department of Astronomy, Peking University, China email: x.liu@pku.edu.cn \\ ${ }^{3}$ Department of Astronomy, Beijing Normal University, China
}

\begin{abstract}
We present LAMOST observations and radial velocity measurements of about 1500 planetary nebulae (PNe) in M31 and M33. Most of the PNe are previously known, but 36 of them are newly discovered in the outskirts of M31, and the furthest one has a projected distance larger than $50 \mathrm{kpc}$ away from the galaxy center. Eighteen objects in the area of M33 are probably newly discovered PNe, and quite a few of them are associated with previously known clusters. For all the $1500 \mathrm{PNe}$, homogeneous radial velocities are measured from the LAMOST spectra, with a typical uncertainty of a few $\mathrm{km} \mathrm{s}^{-1}$.
\end{abstract}

Keywords. planetary nebulae: general; galaxies: kinematics and dynamics

\section{Introduction}

The Guo Shoujing telescope (LAMOST) collects low resolution ( $\mathrm{R} \sim 1800)$ fibre spectra of 4000 objects simultaneously in a sky area of 20 square degrees. The large number of fibres and wide field of view make it particularly suitable to search for planetary nebulae $(\mathrm{PNe}$ ) as well as other special objects (e.g. supergiants, H II regions, globular clusters) in the M31 and M33 galaxies. PNe exhibit prominent emission lines (e.g. [O III]

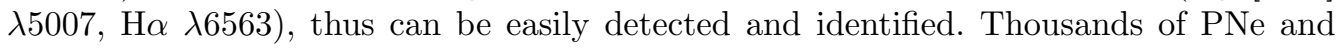
candidates in the area of M31 and M33 have been targeted by LAMOST in both the commissioning $(9 / 2009-9 / 2011)$ and the survey stage $(10 / 2011-6 / 2017)$. The main scientific goals include (1) Validating wavelength calibration of LAMOST spectra; (2) Searching for new PNe, especially in the out skirts of the galaxies; (3) Obtaining accurate and homogeneous measurements of radial velocities and emission line fluxes of a large sample of PNe to study stellar populations, kinematics and chemistry of M31 and M33. With the commissioning data, Yuan et al. (2010) discovered 36 PNe in the outskirts of M31. In this work, we present results from observations carried out in the survey stage.

\section{PN identifications and radial velocity measurements}

A cross-identification with the emission line object catalog of Merrett et al. (2006) yields 1756 observations of 1351 common objects. Comparison of [O III] $\lambda 5007 \mathrm{mag}-$ nitudes deduced from the LAMOST spectra with those of Merrett et al. shows good correlation, indicating that flux calibration of LAMOST spectra is reliable. The differences in [O III] magnitudes of individual PNe have a mean and standard deviation of 0.17 and $0.58 \mathrm{mag}$, respectively. Based on those common objects, a criterion is defined in the $\mathrm{F}(5007) / \mathrm{F}(4861)$ - [O III $]$ magnitude plane to distinguish $\mathrm{PNe}$ and contaminations from other emission line objects, e.g. H II regions. 

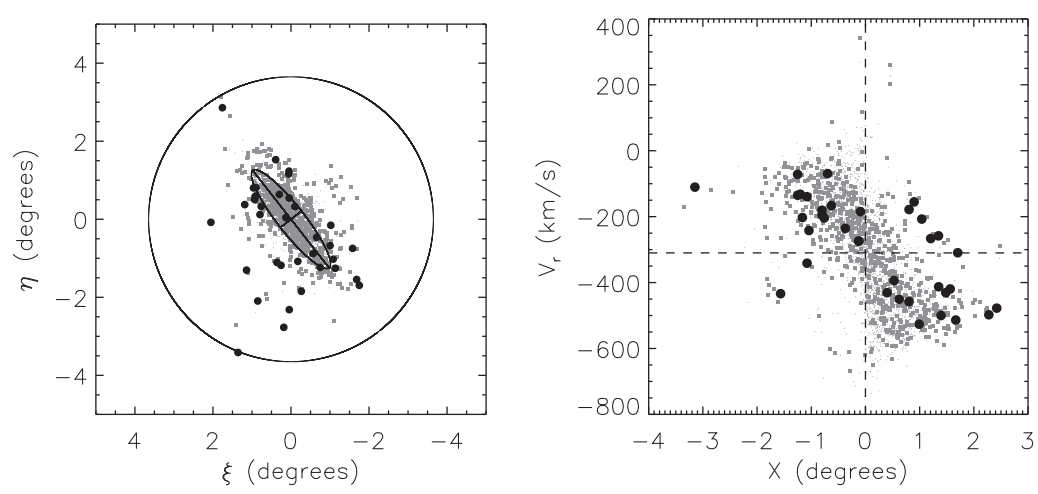

Figure 1. Left: Spatial distribution (Left) and P-V digram (Right) for newly discovered (black) and previously known (grey) PNe of M31. Previously known PNe that have LAMOST observation are shown with squares, while those do not have LAMOST observations are shown with small dots. For the left panel, the circle corresponds a projected distance of $50 \mathrm{kpc}$ to the M31 center. The ellipse has a semi-major radius of $95.3 \mathrm{arcmin}$, an inclination angle of $77 .{ }^{\circ} 5$ and a position angle of $38 .^{\circ} 1$. For the right panel, the abscissa is the projected position along the major axis of M31. The horizontal dashed line denotes a radial velocity of $-310 \mathrm{~km} \mathrm{~s}^{-1}$.

After inspecting literatures and the SIMBAD database, we confirm that $36 \mathrm{PNe}$ are newly discovered around M31. Their spatial distribution and locations in the positionvelocity $(\mathrm{P}-\mathrm{V})$ diagram are shown in Figure 1 . Two of the newly discovered PNe have moderate continnum in their spectra, for which one is possibly contaminated by a nearby Wolf-Rayet star, and the other is associated with globular cluster candidate. The furtherest newly discovered PN has a projected distance larger than $50 \mathrm{kpc}$ away from the M31 center, and probably associated with the giant stellar stream. In the sky area of M33, eighteen objects are probably PNe newly discovered, 5 of them are isolated with very weak continuum in their spectra, while the other 13 are associated with previously known globular clusters or Wolf-Rayet stars.

A comparison of radial velocities deduced from [O III] $\lambda 5007$ lines of LAMOST spectra with those of Merrett et al. yields a mean difference of $-10.0 \mathrm{~km} \mathrm{~s}^{-1}$, and a standard deviation of $20.3 \mathrm{~km} \mathrm{~s}^{-1}$. While a comparison with Halliday et al. (2006) yields a mean difference of $-3.2 \mathrm{~km} \mathrm{~s}^{-1}$ and a standard deviation of $7.0 \mathrm{~km} \mathrm{~s}^{-1}$. For objects with [O III] magnitudes brighter than $23 \mathrm{mag}$, the standard deviation reduces to $5.0 \mathrm{~km} \mathrm{~s}^{-1}$. Radial velocities for $1500 \mathrm{PNe}$ in M31 and M33 are deduced from LAMOST spectra, with a typical uncertainty of a few $\mathrm{km} \mathrm{s}^{-1}$. Fluxes of prominent emission lines of those PNe are also measured.

\section{References}

Halliday C., Carter D., Bridges T. J., Jackson Z. C., Wilkinson M. I., Quinn D. P., Evans N. W., Douglas N. G., Merrett H. R., Merrifield M. R., Romanowsky A. J., Kuijken K., \& Irwin M. J. $2006 M N R A S, 369,97$

Merrett H. R., Merrifield M. R., Douglas N. G., Kuijken K., Romanowsky A. J., Napolitano N. R., Arnaboldi M., Capaccioli M., Freeman K. C., Gerhard O., Coccato L., Carter D., Evans N. W., Wilkinson M. I., Halliday C., \& Bridges T. J. 2006 MNRAS, 369, 120

Yuan H.-B., Liu X.-W., Huo Z.-Y., Zhang H.-H., Zhao Y.-H., Chen J.-J., Bai Z.-R., Zhang H.T., Zhang H.-W., García-Benito R., Xiang M.-S., Yan H.-L., Ren J.-J., Sun S.-W., Zhang Y., Li Y.-P., Lu Q.-S., Wang Y., Ni J.-J., \& Wang H. 2010, Research in Astronomy \& Astrophysics, 10, 599 